\title{
Exploration Technologies for Operations
}

\author{
Ernest E. Smith ${ }^{1}$, David J. Korsmeyer ${ }^{2}$, Vern F. Hall ${ }^{3}$, Jessica Marquez ${ }^{4}$, David Iverson ${ }^{5}$, Jay Trimble ${ }^{6}$, Richard M. \\ Keller $^{7}$, Jeremy Frank ${ }^{8}$, John Chachere ${ }^{9}$, William J. Clancey ${ }^{10}$ \\ NASA Ames Research Center, Moffett Field, Ca, 94035, USA
}

\begin{abstract}
Although the International Space Station (ISS) assembly has been completed, the Operations support teams continue to seek more efficient and effective ways to prepare for and conduct the ISS operations and future exploration missions beyond low earth orbit. This search for improvement has led to a significant collaboration between the NASA research and advanced software development community at NASA Ames Research Center and the Mission Operations community at NASA Johnson Space Center. Since 2001, NASA Ames Research Center has been developing and applying its advanced intelligent systems and human systems integration research to mission operations tools for several of the unmanned Mars missions operations. Since 2006, NASA Ames Research Center has also been developing and applying its advanced intelligent systems and human systems integration research to mission operations tools for manned operations support with the Mission Operations Directorate at NASA Johnson Space Center. This paper discusses the completion of the development and deployment of a variety of intelligent and human systems technologies adopted for manned mission operations. The technologies associated with the projects include advanced software systems for operations and human-centered computing. Human-centered computing looks to the processes and procedures that people do to perform any given job, then attempts to identify opportunities to improve these processes and procedures. In particular, for mission operations, improvements are quantified by specifically identifying how a tool can increase a person's efficiency, enhance a person's functional capability, and/or improve the assurance of a person's decisions. The Ames development team has collaborated with the Mission Operations team to identify areas of efficiencies through technology infusion applications in support of the "Plan, Train, and Fly" activities of human-spaceflight mission operations. The specific applications discussed in this paper are in the areas of mission planning systems, mission operations design modeling and workflow automation, advanced systems monitoring, mission control technologies, search tools, training management tools, spacecraft solar array management, spacecraft power management, and spacecraft attitude planning. We discuss these specific projects between the Ames Research Center and the Johnson Space Center's Mission Operations Directorate, and how these technologies and projects are enhancing the mission operations support for the International Space Station. We also discuss the challenges, problems, and successes associated with long-distance and multi-year development projects between the research team at Ames and the Mission Operations customers at Johnson Space center. Finally, we discuss how these technology infusion applications and underlying technologies might be used in the future to support on-board

${ }^{1}$ Project Integration Manager, Intelligent Systems Division (Code TI), NASA Ames Research Center detailed at Johnson Space Center.

${ }^{2}$ Director of Engineering, NASA Ames Research Center (Code R), AIAA Fellow.

${ }^{3}$ AES DTN Project Manager, NASA Johnson Space Center (Code YI)

${ }^{4}$ Human Systems Integration Division (Code TH), NASA Ames Research Center

${ }^{5}$ Computer Engineer, Intelligent Systems Division (Code TI), NASA Ames Research Center

${ }^{6}$ Intelligent Systems Division (Code TI), NASA Ames Research Center

${ }^{7}$ Intelligent Systems Division (CodeTI), NASA Ames Research Center

${ }^{8}$ Intelligent Systems Division (Code TI), NASA Ames Research Center

${ }^{9}$ Senior Computer Scientist and Project Manager, SGT Inc., Intelligent Systems Division (Code TI), NASA Ames

Research Center

${ }^{10}$ Florida Institute for Human and Machine Cognition, Pensacola
\end{abstract}


operations of the crew and spacecraft systems as human exploration expands beyond low earth orbit to destinations in the solar system where communications delays will require more on-board autonomy and planning by the crew. Longer communications delays will require that the ground mission operations support will be primarily strategic in nature, while the tactical level of planning, systems monitoring and control, and failure analysis/isolation/recovery will be the responsibility of both the spacecraft autonomous systems and the crew. Our expectation is that the technologies that are utilized and matured for the ground mission operations teams will ultimately migrate to the on-board spacecraft systems and crew support utilities for our future human exploration programs.

\section{Introduction}

The National Aeronautics and Space Administration (NASA) is posturing itself for the next major step in the exploration of space, and will require the development of many new capabilities including the design of new spacecraft, new launch services, and new processes and tools associated with the mission operations support. The mission operations support includes the planning of the NASA missions, the training of the crew and flight control team, and the mission execution (referred to as "Plan, Train, and Fly"). The specific targets for NASA mission operations beyond the International Space Station (ISS) Program are currently being assessed by the agency and it is clear that NASA will need to infuse new technologies into the new space exploration initiatives. The range and complexity of these exploration missions will require an unprecedented use of automation and robotics in support of human crews. This will require the operations of manned spacecraft in close conjunction with planetary robotic systems.

NASA's current space flight missions are still largely segmented into unmanned missions funded by the NASA's Science Mission Directorate and the human spaceflight missions operated by the Human Exploration and Operations Mission Directorate. Typically the organizations within NASA that operate the unmanned missions are different from the organizations that operated crewed space systems. The mission operations requirements and needs for the robotic missions have been relatively distinct from those for the Space Shuttle and the ISS.

Developing and validating any new exploration spacecraft and its associated infrastructure places requirements on operations design for future exploration missions - as the development of systems during the Constellation Program has shown. Separate mission operations processes - and cultures - have evolved to support manned missions and unmanned/robotic missions, each appropriately geared to the unique challenges of the two classes of missions. ${ }^{1}$ Developing and maturing the advanced software technologies and process innovations that can benefit both the robotic missions and to also benefit crewed missions is not simple or straightforward. NASA Ames Research Center has been working with both human spaceflight and robotic systems communities now for several years developing mission operations tools and system and is helping to bridge the gap between tools for manned and robotic mission operations.

\section{A. Mission Operations Directorate Needs}

In collaboration with the NASA's Astronaut corps, the Johnson Space Center's (JSC) Mission Operations Directorate (MOD) manages and maintains the flight operations of all of NASA's human spaceflights. These include the previous manned spaceflights from Apollo through the Space Shuttle, and now the International Space Station (ISS). The flight operations of the crewed elements of the future exploration initiatives will likely also be managed by MOD, though MOD's involvement in the potential commercial human spaceflight operations is still evolving.

Manned flight operations support for the ISS is provided by the combination of several ground mission control centers around the U.S. and the World, primarily focused through the Mission Control Center (MCC) at Johnson Space Center (JSC) in Houston, Texas. The ISS is supported by both the MCC and the Payload Ops Integration Center (POIC) at Marshal Space Flight Center (MSFC) in Huntsville, Alabama, plus the International Partners control centers including the Mission Control Center in Moscow (MCC-M or TsUP), the European Space Agency (ESA) Columbus Control Center in Germany and the ATV Control Center in France, the Canadian Space Agency (CSA) Control Center in St. Hubert, Canada, and the Japanese Experiment Module (JEM) and HTV Control Centers in Tsukuba, Japan.

With the retirement of the Shuttle in 2011, MOD at JSC has evolved its flight operations support for the ISS to be more efficient in support of the "Plan, Train, and Fly" activities associated with manned flight operations, thereby reducing its manpower requirements beyond what would have been achieved with the Space Shuttle retirement 
alone. One of the techniques implemented for achieving additional efficiencies has been the investment in several technology infusion applications development opportunities as described within this paper.

\section{Intelligent Systems and Human Systems Integration at NASA}

\section{A. Advanced Software Systems for Operations}

NASA Ames Research Center has long been a leader in the development of advanced software technologies and systems for NASA Missions. Through the 1990s this role included leadership of the majority of NASA's automated reasoning and human-centered computing programs. In the first decade of this millennium, Ames leads all of the advanced software research and development projects for the Exploration Systems Mission Directorate. Ames has exercised these responsibilities to provide the Agency with a notable set of software technology "firsts," including the first autonomy software to be flown by NASA on a spacecraft ${ }^{2}$ and the first advanced planning software to daily plan a robotic planetary mission. ${ }^{3}$ Similarly the multiyear partnership with the manned spaceflight operations described in this paper has grown out of a combination of excellent technical work, a focus on NASA's needs and vision of its future, and a portfolio of activities ranging from needs-driven technology development to projectfocused tool development.

The partnership between NASA Ames Exploration Technology Directorate and NASA JSC's MOD has been addressing the significant challenges posed by the agency's long-term operations of manned spacecraft. The knowledge, intelligence, and engineering analysis currently provided by MOD mission controllers will need to be automated and accompany the astronauts on future missions to both the ISS and exploration destinations. ISS is now the Agency's premier test-bed for vehicle systems, and mission operations will need to be more adaptable for varied mission scenarios. An ability to rapidly and dependably develop and modify software could provide MOD the means to alter system capabilities on the fly. Following current practices, software modifications to space-based and flight control systems can take in the months or years to make. To modify capabilities between and during missions, revolutionary software development approaches are needed - new approaches that, in the tens of minutes, can result in effective and dependable modifications. Like MOD, in order to achieve these goals Ames must target an evolutionary path to proving out technical approaches. During this evolution it must validate the value for the crewed mission operations community.

\section{B. Human-Centered Computing}

The lessons learned from NASA Ames developers and the experience they had creating tools for the Jet Propulsion Laboratory's Mars planetary missions and the past years of collaboration with MOD have defined a framework for how Ames and JSC determine what opportunities exist for intelligent systems applied to the crewed spacecraft operations for NASA. Principally the discussion of new technologies and tools needs to be framed in a manner that identifies and emphasizes the value to the MOD operational flight controller. It is not about replacing 'man with a machine', but about complementing and augmenting the flight controller or operator to do a better job. This overall methodology and approach is often referred to as human-centered computing.

Human-centered computing looks to the processes and procedures that people do to perform any given job, then with these understanding attempts to identify opportunities to improve these processes and procedures. In particular, for mission operations, improvements are quantified by specifically identifying how a tool can increase a person's efficiency, enhance a person's functional capability, and/or improve the assurance of a person's decisions.

A human-centered computing strategy contains the following essential elements:

- Deploy personnel to mission centers to work with the NASA customer(s) to understand the exact nature of their current work and future challenges that may be amenable to software solutions.

- Scout for all relevant approaches or technologies that may address the customer's needs.

- Identify technology gaps left by current software capabilities to seed new research and development.

- Simultaneously conduct carefully-targeted research and development to address the gaps on an ongoing basis.

- Evaluate and compare competing results, working closely with the customer to determine the strengths and weaknesses and the cost-benefit of the each candidate solution and improve it on this basis.

This above strategy worked exceptionally well for infusing advanced technologies in the Mars robotic missions with Spirit and Opportunity rover, the Phoenix Lander, and the upcoming Mars Science Laboratory. It has proven to work equally well for the human spaceflight community operating Space Station and the Shuttle. 


\section{Advanced Mission Operations Development Projects}

At the advent of the Constellation Program, Ames and JSC began discussions about how to leverage each other's strengths and capabilities, and established a set of initial projects that addressed outstanding needs within MOD. These projects first started in 2006 and have progressed to several full-scale projects incrementally delivering operational flight controller tools through calendar year 2013. All of these mission operations development projects are considered relevant to increasing the efficiency of the mission operations flight control team, and supported the goal of overall operations manpower reductions while ensuring the high level of safety for mission operations. Even with the cancellation of the Constellation Program, the need for innovative and efficient mission operations remains. The continuation of ISS operations until at least 2024 means that operational efficiency is a driving factor in keeping costs of the ISS flying minimum. A synopsis status of some of the projects is presented below.

\section{A. Mission Planning System Enhancements}

The MOD Operations Planning Timeline Integration System (OPTimIS - previously referred to as the Next Generation Planning System (NGPS)) project aims to develop one planning system and process for all programs to leverage with technologies to improve and simplify mission planning for all MOD teams. Key components of the OPTimIS software suite includes Score (planning software interface) and Plan Repository (online database of plans), both developed by the Human Computer Interaction (HCI) Group at Ames. These software products incorporate enhanced scheduling capabilities based on the Ames Scheduling and Planning Interface for Exploration (SPIFe) tools. The other OPTimIS components (e.g., WebAD \& Viewer) were developed by JSC MOD contractors. The collaboration between MOD, their contractors, and the Ames technologists will consolidate many of the disparate planning tools being used today. ${ }^{4}$ This is envisioned to have a significant impact on the overall efficiency of the planning efforts for ISS. Score has been operationally deployed and in use by flight controllers since 2012, while Plan Repository has been in use since 2014.

ISS Ops Planners are now able to use Score to plan for the entirety of a single expedition, six months worth of data integration and planning with one single software tool. Previously, this planning required several tools, and a significant portion of the flight controllers' time was spent "translating" data from one tool to another. With Score, Ops Planners can lay out a first cut of all the required activities to be completed during one mission. Next, the flight controllers can parse weekly plans and refine them, ensuring that all operational constraints are met. Score is essential to helping users define and identify all the operational constraints included in a plan for six astronauts. The complex set of constraints is checked within Score and any violation is flagged. If possible, Score suggests possible ways of resolving the violation. Once the plan is refined, the rest of the mission control team, including the international partners, review the plan (through either the On-Board Short Term Plan Viewer/OSTPV or the Viewer) and request changes to the plan as needed. Once these change requests are approved, Score facilitates their implementation in the plan and auto-completes the requested changes. Another timesaving task that Score enables is cross-product linking with other tools across mission control, such as software procedure tools.

Plan Repository was developed to make possible two new, key features: seamless version control of plans and real-time collaboration planning. Previously, plan version control was managed through individual files; with Plan Repository, all plans reside in a server and edits are tracked and saved automatically for users. Once a particular version of the plan is ready for publication, it is tagged with a label so that others in the mission control team can access. Once plans were accessible through a server, we were able to provide users the ability for real-time collaboration planning. This meant that Ops Planners, within Mission Control Center and around the world, could access the same plan, make real-time edits and share those edits with everyone else, eliminating the tedious and ineffective process of exchanging emails with plan changes.

\section{B. Mission Operations Design Modeling and Workflow Automation}

OCAMS (Orbital Communication Adapter Management System) is a practical engineering application of multiagent systems technology, using the Business Redesign Agent-based Holistic Modeling System (BRAHMS) modeling and simulation tool. ${ }^{5}$ The BRAHMS system combines models of systems (e.g., robots, tools, software) with models of people communicating and moving in a simulated geographic space, revealing how interactions of people, facilities, and tools are productive or gaps that may occur in capabilities and procedures. BRAHMS simulations can be converted into a runtime system in which software agents automate and mediate work flow operations and communications among people and systems.

The project began in November 2006, leading to a completed workflow automation system that is currently being used by OCA Officers in the MPSR backroom supporting the ISS. The OCA Officer is responsible for uplinking and down-linking all files to and from the ISS, based on scheduled operations and requests from ground support. The OCA Officer mirrors, archives, and forwards files to support personnel and documents this work in flight notes and logs. The Ames OCAMS project team has developed the agent-based OCAMS to perform this entire

4

American Institute of Aeronautics and Astronautics 
process by using the Simulation-to-Implementation Engineering method. ${ }^{6}$ First they simulated the OCA Officer's work practices to identify possible process improvements. Using statistics generated from this simulation model and collaborative design with the OCA Team at JSC, they then developed an agent-based workflow system that supports the redesigned and improved OCA work process. With the OCAMS system, the time spent by the OCA Officer processing files uplinked and down-linked to the Space Station has been sufficiently reduced to enable merging this console position, thereby reducing the manpower required to support this function. This project was completed in 2013 and the OCAMS application continues to be heavily used within the MCC at JSC, providing 24/7 support to the ISS flight control team.

\section{Advanced Systems Monitoring}

The Anomaly Monitoring Inductive Software System (AMISS) is an Ames developed health monitoring software application that compares current system data with data from previous nominal system operations. AMISS is based on the Ames developed Inductive Monitoring System (IMS), and applies data mining techniques to archived telemetry to establish a baseline of normal behavior for groups of data parameters from the monitored system. AMISS then uses that baseline to identify off-normal behavior in real-time telemetry, potentially alerting the mission operations team to problems prior to any caution and warning annunciation for the system. Any deviations from normal baseline behavior will be indicated by AMISS with a non-zero "distance" from nominal. Information is also provided on which data parameters are contributing to the off-nominal readings to help identify the source of the anomaly. ${ }^{7}$

In 2006, Ames delivered AMISS based tools to JSC that allow mission operations users to retrieve archived mission data and run the data offline on AMISS to both "train" the tool on nominal data and to execute the "monitoring" feature. This capability was tested on several ISS Control Moment Gyroscope (CMG) data sets, including data collected during some significant CMG malfunctions. AMISS successfully detected anomalies in CMG behavior in these data sets, sometimes several hours before malfunctions were detected by current MCC systems. These promising results prompted JSC to establish a 2007-08 task to deploy the tool within the MCC environment for evaluation and use in real time by the on-console flight control team. The AMISS tool has been integrated with the MCC real time data system and deployed on the mission control consoles in the ISS control room to provide real time CMG monitoring. In addition, AMISS has been augmented with fault detection routines that will automatically detect and identify some common CMG faults to assist controllers in diagnosis and recovery activities. AMISS has also been applied to the monitoring of the ISS External Thermal Control System (ETCS) subsystems.

The AMISS software has been updated to use the ODVEC (Outlier Detection Via Estimating Clusters) monitoring algorithm. That's an extension of IMS that compares the real-time data to a larger sample of nominal data using an $\mathrm{N}$ nearest neighbor's technique. The CMG, RGA (rate gyro assemblies), and ETCS models were updated for ODVEC monitoring and certified in 2012 for console use. AMISS has also been set up in the MCC MER (Mission Evaluation Room) for off-line data analysis use.

\section{Mission Control Technologies}

During the course of multiple human space flight programs, MOD mission operations systems have been built as a collection of monolithic software applications. Each application serves the needs of a specific user base associated with a discipline or functional role. Designed to accomplish specific tasks, each application embodies specialized functional knowledge and has its own data storage, data models, programmatic interfaces, user interfaces, and customized business logic. In effect, each application creates its own walled-off environment. While individual applications are sometimes reused across multiple missions, it is expensive and time consuming to maintain these systems, and both costly and risky to upgrade them in the light of new requirements or modify them for new purposes. It is even more expensive to achieve new integrated activities across a set of monolithic applications.

These problems impact the life-cycle cost (especially design, development, testing, training, maintenance, and integration) of each new mission operations system. They also inhibit system innovation and evolution. This in turn hinders NASA's ability to adopt new operations paradigms, including increasingly automated space systems, such as autonomous rovers, autonomous onboard crew systems, and integrated control of human and robotic missions.

In order to achieve NASA's vision of affordably and reliably, consideration has been given to build mission control systems that overcome the problems inherent in systems of monolithic applications. Two possible keys to the solution are modularity and interoperability. Modularity would increase extensibility, reusability, and maintainability. Interoperability would enable composition of larger systems out of smaller parts, and make possible 
the construction of new integrated activities that tie together, at a deep level, the capabilities of many of the components. Modularity and interoperability together contribute to flexibility.

The Mission Control Technologies (MCT) Project, ${ }^{8}$ a collaboration of multiple NASA Centers led by Ames, is building a framework (based upon the open-source Eclipse software) to enable software to be assembled from flexible collections of components and services. MCT was deployed in the MCC Operations Test Facility (OTF) for several years, shadowing ISS mission operations. It was also deployed into the MCC to expand the user base that could evaluate the cyclic deliveries of this capability and to develop, gather, and analyze measurements to evaluate the performance and usability of MCT, from a flight controller's perspective. This move also enhanced the ability to define and analyze the proper engineering metrics - performance, lines of code, and the potential cost savings.

Unfortunately, due primarily to budget reductions, the MCT implementation within the MCC was cancelled at the end of fiscal year 2012. Instead, the MOD team will rely on legacy applications upgraded in an internal project referred to as MDT (MOD Display Tool), plus the user environment improvements inherent in the MOD upgrade of the MCC, referred to as MCC21 (21 $1^{\text {st }}$ Century MCC). However, influences of MCT can be seen in the MDT project. There were four capabilities of the MCT project that were recognized as also being needed for the the MDT project. These MCT-Derived requirements and goals for MDT included: 1) the requirement to have the capability to re-use existing operational display content and layout information from all existing legacy tools, 2) the goal to reduce the total resources required to build, verify and maintain new plan/train/fly displays, 3) the goal to have the capability for on-console personnel to build, verify and test new displays during real-time operations and simulations, and 4) the goal to have the capability to display any combination of data, graphics and plots/graphs on the same display; i.e., within the same window.

\section{E. Search Tools}

The Cross-MCC Search project (XSearch) focused on improving MOD access to and retrieval of critical information required to monitor, control, and manage ISS and Space Shuttle. ${ }^{9}$ While much of this information (in the form of notes, change requests, action item lists, procedures, documentation, etc.) was accessible using a patchwork of disconnected tools and databases, this project built a unified search capability across these data sources thereby presenting a unified single Web-based interface for all MOD flight controllers. In addition, the system identifies cross-referenced and other relevant information that flight controllers might otherwise overlook.

The XSearch development, working closely with MOD Flight Controllers, began in January 2006 and the first version was deployed in the MCC environment during June 2008 to enable search and cross-referencing across three key mission control data sources (mission Flight Notes, Anomaly Reports and Chits). Eight subsequent versions were delivered to MOD, each folding new data sources. Flight controllers can search a total of 15 sources simultaneously with the current version, which was delivered in September 2010. This tool continues to be used by both the Flight Control Team and the MER engineers.

\section{F. Solar Array Management}

As the construction of the ISS was being completed, the full complement of solar arrays was added to provide the power required to support additional modules on the larger station. These new arrays had more freedom to articulate, enabling better tracking of the sun and thus increased power production. However, these arrays also had more complex constraints that limit the range of safe orientations, due to structural loads, contamination concerns, and thermal impacts. These limitations on safe array orientations impact power generation, which requires MOD flight controllers to constantly balance multiple complex constraints against ISS power needs. The increased complexity does not only impact pre-planning activities, but has an even more acute effect on real-time operations, in particular when handling unexpected events or changes in operations plans.

The Solar Array Constraint Engine (SACE) project has developed a tool that provides intelligent decisionsupport capabilities to ISS power systems flight controllers, to assist them with the task of planning and executing solar array operations in a safe, sustainable, and effective manner. ${ }^{10}$ SACE provides situational awareness, orientation evaluation and optimization, and array operations planning functionality to flight controllers. The SACE tool is built on the EUROPA engine, which provides constraint management and reasoning, decision-support and planning.

The SACE tool enables simple solar array plan generation. SACE includes: (1) separation of plan configuration and generation into separate phases, so that the user can modify the configuration before proceeding with plan generation; (2) improved algorithm for consolidating constraints and user restrictions during the solution of configurations that need to be merged during plan generation; (3) visual indication to the user of configurations merged during planning; (4) reporting of orbital-average power availability for the generated plan, taking into 
consideration the actual configuration/orientation of the arrays during each orbit; (5) displaying of eclipse and insolation timelines; and (6) support for custom constraints representing complex, unique array position restrictions.

SACE is a fully delivered capability for enhancing the flight control real-time monitoring of the ISS solar arrays and associated constraints management. Development continues to certify the flight planning capability for solar array operations.

\section{G. Power Management}

The ISS flight control team currently utilizes a complex suite of tools to plan and analyze ISS power management. The Power Planning and Analysis Tool (PLATO) project was a collaboration project that consolidated the existing ISS power planning tasks into a single application with an easily manipulated interface and facilitates the sharing of power-related data with other flight control disciplines. ${ }^{11}$ It would still maintain the robust capability of the current power analysis toolset, allowing the specialized flight controllers to delve into the underlying models and modify the analysis parameters for ISS. The HCI Group at Ames developed PLATO, leveraging the SPIFe and Eclipse framework to integrate new power planning models as well as incorporated existing MOD power planning analysis software tools. PLATO has been operationally deployed and in use by ISS flight controllers since 2013.

The basic function of PLATO is to determine (through power analysis engines) the amount of power available for ISS and the amount of power to be consumed for that week. Any difference between availability and consumption levels requires power management by the Power Resource Officer (PRO, specialist flight controller). Through PLATO, all required data inputs are automatically collected, properly translated, and an initial power planning analysis project is created. This initial step, while apparently simple, was an extremely time consuming task the power planning flight controllers had to complete before even starting their analysis. They spent much time translating the inputs (ranging from a Word or Excel document to text files describing attitude maneuvers) into a file type that their analysis models could interpret. PLATO converts all the inputs, allowing users to spend time on the more challenging task of power management. PLATO also integrated all the analysis data between several power analysis engines, another timesaving task resulting from an integrated software system. Once an initial analysis is completed (which includes running five analysis engines), the flight controllers need to ensure that none of the operational constraints are violated. PLATO flagged violations through one interface and facilitated creating "power-downs". Power-downs are planned events for ISS that are necessary when there are discrepancies between ISS power availability and consumption. Finally, PLATO automatically generated all the required data that is shared to the rest of the mission control team.

\section{H. Attitude Planning}

The ISS Attitude Determination and Control Officer (ADCO) has overall responsibility for the integration of all Guidance, Navigation and Control, propulsion and Interim Control Module activities (including monitoring CMGs). ADCO works in partnership with Russian controllers to manage the station's orientation, controlled by the onboard Motion Control Systems. This position also plans and calculates future orientations and maneuvers for the station. The position of the space station is one of the most fundamental components for all ISS operations. The ADCO Planning Exchange Tool (APEX) was developed for ADCOs to streamline their existing manual and time-intensive attitude planning process, enabling a more automated application that interfaces with existing products and allows the ADCO to produce accurate products and timelines more efficiently. APEX has been operationally deployed and in use by ISS flight controllers since 2012.

Both Russian and US flight controllers manage ISS's position and attitude maneuvers. Each of these maneuvers needs to be coordinated and approved by both. Before APEX, ADCO exchanged emails, which were manually created. APEX facilitated processing of these messages, computer-generating the exchange files, and hence removing the possibility of incorrect typing. APEX also tracks the state of approvals, offloading this task from the ADCO, who can then focus on the more difficult task of determining the appropriate ISS maneuvers. APEX, developed also by the HCI Group at Ames, leveraged the SPIFe framework. This made planning and replanning of the ADCO events a simple timeline edit and then APEX generated all the necessary exchange files. Finally, ADCOs are able to access information stored in Plan Repository as well as interact with OPTimIS to submit plan change requests.

\section{Future Operations Support}


Human centered technology derived mission operations and flight control applications will ultimately be needed in the future to support on-board operations of the crew and spacecraft systems as human exploration expands beyond low earth orbit to destinations in the solar system where communications delays will require more on-board autonomy and planning by the crew. Longer communications delays will require that the ground mission operations support will be primarily strategic in nature, while the tactical level of planning, systems monitoring and control, and failure analysis/isolation/recovery will be the responsibility of both the spacecraft autonomous systems and the crew. Our expectation is that the technologies that are utilized and matured for the ground mission operations teams will ultimately migrate to the on-board spacecraft systems and crew support utilities for our future human exploration programs. These advanced exploration technologies are being assessed by the Ames Research Center technologists for applications to the Orion program and other advanced exploration possibilities.

\section{Conclusion}

NASA's Ames Research Center and Johnson Space Center have worked together to apply intelligent systems to mission operation tools and systems. This work is critical to the Agency, ISS and possibly any future human exploration programs. Improving the capacity of NASA's main manned mission operations teams to handle more operations per controller, enhancing the capabilities of those teams to handle complex decisions in a timelier manner, and increasing the available knowledge to the flight controller to make safer decisions are the critical motivations for this work.

This paper presents the culmination of technology infusion applications development projects intended to greatly enhance how Mission Operations are performed for human spaceflight within NASA. These efforts enhance the current MOD mission operations support of the International Space Station, and these same mission operations applications technologies may one day enhance the spacecraft and crew support capabilities with ground-proven automated and autonomous systems for our future human exploration missions. 


\section{Appendix A}

\section{Acronym List}

\begin{tabular}{|c|c|}
\hline AES & Advanced Exploration Systems \\
\hline ADCO & Attitude Determination and Control Officer \\
\hline AMISS & Anomaly Monitoring Inductive Software System \\
\hline AMO & Autonomous Mission Operations \\
\hline APEX & ADCO Planning Exchange Tool \\
\hline ARC & Ames Research Center \\
\hline ATV & Autonomous Transfer Vehicle \\
\hline BRAHMS & Business Redesign Agent-based Holistic Modeling System \\
\hline CMG & Control Moment Gyroscope \\
\hline ConFRM & Constraints and Flight Rules Management \\
\hline CPS & Consolidated Planning System \\
\hline CSA & Canadian Space Agency \\
\hline ESA & European Space Agency \\
\hline ETCS & External Thermal Control System \\
\hline EUROPA & Extensible Universal Remote Operations Planning Architecture \\
\hline HEFT & Human Exploration Framework Team \\
\hline HTV & H-II Transfer Vehicle \\
\hline IMS & Inductive Monitoring System \\
\hline IPS & Integrated Planning System \\
\hline ISS & International Space Station \\
\hline JEM & Japanese Experiment Module \\
\hline JSC & Johnson Space Center \\
\hline KSC & Kennedy Space Center \\
\hline LaRC & Langley Research Center \\
\hline MCC & Mission Control Center \\
\hline MCC-M & Mission Control Center in Moscow \\
\hline MCT & Mission Control Technologies \\
\hline MOD & Mission Operations Directorate \\
\hline MSFC & Marshal Space Flight Center \\
\hline MSL & Mars Science Laboratory \\
\hline NASA & National Aeronautics and Space Administration \\
\hline NEOs & Near-Earth Objects \\
\hline NGPS & Next Generation Planning System \\
\hline OCA & Orbital Communication Adapter \\
\hline OCAMS & Orbital Communication Adapter Management System \\
\hline OPTimIS & Operations Planning Timeline Integration System \\
\hline OSTPV & On-Board Short Term Plan Viewer \\
\hline OTF & Operations Test Facility \\
\hline PLATO & Power Planning and Analysis Tool \\
\hline POIC & Payload Ops Integration Center \\
\hline SACE & Solar Array Constraint Engine \\
\hline SPIFe & Scheduling and Planning Interface for Exploration \\
\hline STAR & Scheduling, Training Administration, and Records \\
\hline STP & Short Term Plan \\
\hline TAMS & Training Administration Management System \\
\hline XSearch & Cross Search \\
\hline
\end{tabular}




\section{Acknowledgments}

The Authors wish to acknowledge all of the multiple project leads and team members at Ames, JSC, MSFC, KSC, JPL, and LaRC for the above-mentioned projects. We would also like to acknowledge the support of the Mission Operations Directorate flight controllers, trainers, facilities developers and the Mission Operations Directorate management team for their collaboration and support for these projects. Their work is changing the way that mission operations is performed for the International Space Station and enabling NASA to be ready to fully support future human exploration programs.

\section{References}

${ }^{1}$ Mishkin, A., Lee, Y., Korth, D., and LeBlanc, T., "Integrated Human-Robotic Missions to the Moon and Mars: Mission Operations Design Implications,” IEEEAC paper \#1400, Version 2, Updated November 2, 2006.

${ }^{2}$ Muscettola, N., Nayak, P., Pell, B., and Williams, B., "Remote agent: To boldly go where no AI system has gone before," Artificial Intelligence, 103(1-2):5 - 48, 1998.

${ }^{3}$ Bresina, J., Jónsson, A., Morris, P., and Rajan, K., "MAPGEN: Mixed Initiative Planning and Scheduling for the Mars '03 MER Mission," International Symposium on AI and Robotics in Space, Nara, Japan, 2003.

${ }^{4}$ Frank, J., Morris, P.H., Greene, J., Hall, T., "The Challenge of Evolving Mission Operations Tools for Manned Spaceflight," 9th International Symposium on Artificial Intelligence, Robotics, and Automation for Space, Los Angeles, CA, 2008; Clement, B., Barreiro, J., "Spatial Planning for International Space Station Crew Operations," Proceedings of the International Symposium on Artificial Intelligence, Robotics and Automation in Space, 2010.

${ }^{5}$ Clancey, W.J., Sachs, P., Sierhuis, M., and van Hoof, R. "Brahms: simulating practice for work systems design," Int. J. Human-Computer Studies, 49, 831-865, 1998.

${ }^{6}$ Clancey, W. J., Sierhuis, M., Seah, C., Buckley, C., Reynolds, F., Hall, T., Scott, M. "Multi-agent simulation to implementation: A practical engineering methodology for designing space flight operations.” In A. Artikis, G. O'Hare, K. Stathis, \& G. Vouros (Eds.), Engineering Societies in the Agents' World VIII. Lecture Notes in Computer Science Series, Volume 4870. Heidelberg Germany: Springer, 2008, pp. 108-123.

${ }^{7}$ Iverson, D. L., "Data Mining Applications for Space Mission Operations System Health Monitoring", Proceedings of the SpaceOps 2008 Conference, ESA, EUMETSAT, AIAA, Heidelberg, Germany, May 2008.

${ }^{8}$ Trimble, J., Crocker, A., "A Flexible Evolvable Architecture for Constellation Mission Systems User Applications," SpaceOps 2008, Heidelberg Germany, May 12-16, 2008.

${ }^{9}$ Keller, R.M., Wolfe, S.R., Windrem, M.N., and Berrios, D.C., "XSearch: A System for Searching and Interrelating NASA Mission Operations Data," SpaceOps 2008 Conference, Heidelberg, Germany, May 2008.

${ }^{10}$ Reddy, S.Y., Ai Chang, M., Iatauro, M., Kurklu, E., Boyce, M., Frank, J., Jonsson, A., "Planning and Monitoring Solar Array Operations on the International Space Station," Proceedings of the Workshop on Planning and Scheduling Applications, in conjunction with the International Conference on Automated Planning, 2008.

${ }^{11}$ Barreiro, J., Jones, G., Schaffer, J., "Peer-to-peer Planning for Space Mission Control," Proceedings of the 6th International Workshop on Planning and Scheduling for Space, (IWPSS) Pasadena, CA., 2009; Aghevli, A., Bachmann, A., Bresina, J.L., Greene, J., Kanefsky, R., Kurien, J.,McCurdy, M., Morris, P.H., Pyrzak, G., Ratterman, C., Vera, A., Wragg. S., "Planning Applications for Three Mars Missions with Ensemble," 5th International Workshop on Planning and Scheduling for Space. (IWPSS) Baltimore, MD, 2007.

${ }^{12}$ Barreiro, J., Chachere, J., Frank, J., Bertels, C., Crocker, A., "Constraint and Flight Rule Management for Space Mission Operations," Proceedings of the International Symposium on Artificial Intelligence, Robotics and Automation in Space, 2010. 